\title{
Restoration technology of the video under snow
}

\author{
Xiaomeng Zhou, Yan Piao*, Ge Shi \\ Electronic and information engineering institute, Changchun University of Science and Technology, \\ Changchun, China \\ piaoyan66@126.com
}

Keywords: Symmetric frame differencing; Snow removal; Fog removal; Image restoration

\begin{abstract}
The snow in video images affects image quality and blurs key objects in the video image. It affects the ability of computer automatic detection, recognition and target tracking. First of all, we can use the improved symmetrical frame differencing method to detect and remove snow. If the snow is intensive, we will remove snow fog to improve the result of restoration. The simulation results show that this method can effectively remove the snow in the video image, it can provide a feasible method for clearness of bad weather.
\end{abstract}

\section{Introduction}

Snow is unstable, its visual effect is very complex. Because of the characteristics of randomness and fast motion of spatial distribution of snow, it makes removing snow in the video image difficult. Garg and Nayar [1, 2] of the Columbia University proposed the method to remove rain by optical model, assuming that the speed and direction is the same, it is not ideal to remove rain. Barnum[3, 4] et al introduced snow and global characteristics, and put forward the method of removal using frequency domain characteristics and this method needs higher falling speed of rain and snow. $\mathrm{Xu}[5]$ used the method of defogging method to remove snow in the video, but the method will leave much snow track, but the image of the object boundary is fuzzy. In this paper, using improved symmetrical frame differencing to detect the snow in the image, according to the pixel brightness relations to remove snow in the image, and then by the defogging method to remove snow. It can increase the clarity of the image, and the experimental results show the effectiveness of the algorithm.

\section{The physical properties of snow}

Falling snow will produce a blurred effect, the snow is like the snowline. Assuming that the exposure time is $\mathrm{T}$, in order to determine the pixel intensity by the snowflake effect, we need to calculate the optical density of the whole time T. Assuming that $\tau$ stands for the time which pixel passed the snow. Because it is much shorter than $\mathrm{T}$, so the intensity of light $I_{d}$ is jointly composed of optical density optical density $E_{b g}$ and background of the snow $E_{d}$ :

$$
I_{d}=\int_{0}^{\tau} E_{d} d t+\int_{\tau}^{T} E_{b g} d t
$$

If the background is slowly moving or stationary, during the exposure time of $\mathrm{T}$, the above formula can be simplified to:

$$
I_{d}=\tau E_{\bar{d}}+(T-\tau) E_{b g}, E_{\bar{d}}=\frac{1}{\tau} \int_{0}^{\tau} E_{d} d t,
$$

$E \bar{d}$ refers to the average optical density of snow. If the pixel is not effected by snow, $I_{g b}=E_{b g} T$

Therefore, a pixel intensity changes caused by the snow $\Delta I$ :

$$
\Delta I=I_{d}-I_{b g}=\tau\left(E_{\bar{d}}-E_{b g}\right)>0
$$




\section{The detection and removal of snow}

The snow is a researching object, which blocks the key objects. The detection and removal of snow is the emphasis of this paper, aiming at the deficiency of previous methods, puts forward the improved symmetrical frame differencing method to detect and remove snow, flow chart of basic method in figure 2.

Fig.2 Flow chart of snow removal

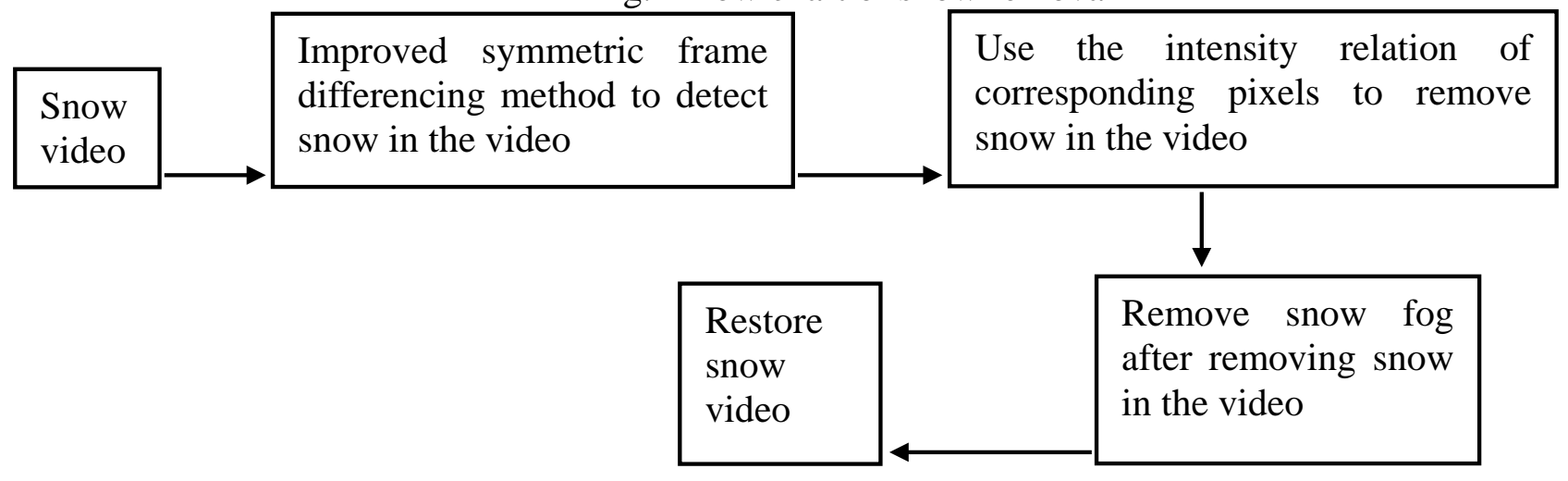

\section{Improved snow detection algorithm}

Symmetrical frame differencing method firstly smoothes three continuous frame images by denoising, then we can use the frame difference method processing and use the $\mathrm{k}$ frame minuses the $\mathrm{k}-1$ frame, we can get the two value image $D_{1}(x, y)$, and then the $\mathrm{k}+1$ frame minuses the $\mathrm{k}$ frame image, get the two value image $D_{2}(x, y)$, and then use $D_{1}(x, y)$ and $D_{2}(x, y)$ to make "and" operation, and then use $D_{2}(x, y)$ and $D_{3}(x, y)$ to make "xor" operation, and then use $D_{3}(x, y)$ and $D_{4}(x, y)$ to make "and" operation we can get a symmetrical frame difference image. Formula is

$$
\begin{aligned}
& D_{1}(x, y)=\left\{\begin{array}{l}
1, \mid f_{k}(x, y)-f_{k}-1(x, y) \geq T \\
0, \mid f_{k}(x, y)-f_{k}-1(x, y)<T
\end{array}\right. \\
& D_{2}(x, y)=\left\{\begin{array}{l}
1, \mid f_{k}+1(x, y)-f_{k}(x, y) \geq T \\
0, \mid f_{k}+1(x, y)-f_{k}(x, y)<T
\end{array}\right. \\
& D_{3}(\mathrm{x}, \mathrm{y})=\mathrm{D}_{1}(\mathrm{x}, \mathrm{y}) \cap D_{2}(x, y) \\
& D 4(\mathrm{x}, \mathrm{y})=\mathrm{D}_{3}(\mathrm{x}, \mathrm{y}) \otimes D_{2}(x, y) \\
& D(\mathrm{x}, \mathrm{y})=\mathrm{D}_{3}(\mathrm{x}, \mathrm{y}) \cap D_{4}(x, y)
\end{aligned}
$$

In the formula, $\mathrm{T}$ is the threshold value set in advance, according to the experience. If the selected $\mathrm{T}$ is too large, then the detection targets may appear larger holes even missing. If the selected $\mathrm{T}$ is too small, there will be a lot of noise. The method is fast, which can better detect the exact outline of the target and reduce the ghost phenomenon.

Based on physical and optical model of the snow, we can use the appropriate constraint and the frame difference method to detect the pixel covered by the snow in each frame. According to the optical model, the snow will produce a positive change in the unit frame time on light intensity. If the background in the three frame is static, and $I_{n-1}$ and $I_{n+1}$ must be equal, and the $\mathrm{N}$ frame in the snow caused by light intensity must satisfy the following constraint:

$$
\Delta I=I_{n}-I_{n-1}=I_{n}-I_{n+1} \geq C
$$

In order to make the removal of snow in video images much better, we improve the above method, we can use continuous five frames to process middle frame, the pixel point satisfies the constraint, or by continuous seven frames for processing middle frame, the pixel point satisfies the constraint:

$$
\begin{aligned}
& \Delta I=I_{n}-I_{n-2}=I_{n}-I_{n+2} \geq C \\
& \Delta I=I_{n}-I_{n-3}=I_{n}-I_{n+3} \geq C
\end{aligned}
$$




\section{The algorithm of snow removal}

Because the gray of pixels covered by snow is greater than the snow which is not covered by snow, so we can use the average corresponding pixel point brightness of available $n-1$ and $n+1$ frame to replace the point, the formula can be expressed as follows:

$$
I_{n}=\left(I_{n-1}+I_{n+1}\right) / 2
$$

In order to make the video images after snow removal look better, we improved the above method. We can use consecutive five or seven frames video image to handle intermediate frames, and then analyze the connection of pixel after improvement, the formula can be expressed as follows:

$$
\begin{aligned}
& \alpha=\frac{I_{n-1}+I_{n+1}}{2} / \frac{I_{n-2}+I_{n+2}}{2} \\
& \boldsymbol{I}_{\boldsymbol{n}}=\left\{\begin{array}{l}
\frac{\boldsymbol{I n}-1+\boldsymbol{I}_{n+1}}{2}, \alpha<1 \\
\frac{\boldsymbol{I n}_{n}-2+\boldsymbol{I}_{n+2}}{2}, \alpha>=1
\end{array}\right.
\end{aligned}
$$

Also we can go to the sort of size corresponding pixel brightness section $n-2, n-1, n+1$ and $n+2$ frame image ,and background pixels is relatively dark, and then use the brightness minimum point to replace the prime point, formula is as follows:

$$
I_{n}=\min \left[I_{n}-2, I_{n-1}, I_{n}+1, I_{n}+2\right]
$$

For the video image after removing the snow, because of darkened snow weather, the image will have snow fog, so we can use the method to remove fog. We can use the defogging methods commonly to enhance the brightness of the image after removing snow.

\section{Analysis of experimental results}

Figure 4 shows the detection of snow, the improved detection method is better than the previous method ,the method can preserve the moving snow, interference the elimination from automobile and building in the picture, the shape and position of detection is more accurate. Figure 5 shows the removal of snow, the previous method is difficult to adapt to moderate snow and heavy snow, the proposed algorithm can remove most of the snow, and there is no obvious distortion situation, before and after removal of snow, the PSNR reaches 34.0343.

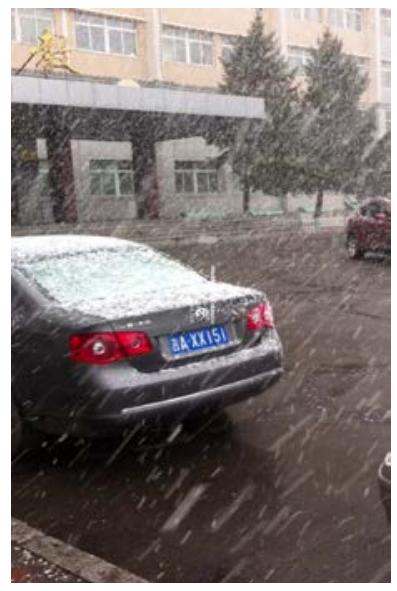

(a) original video frame

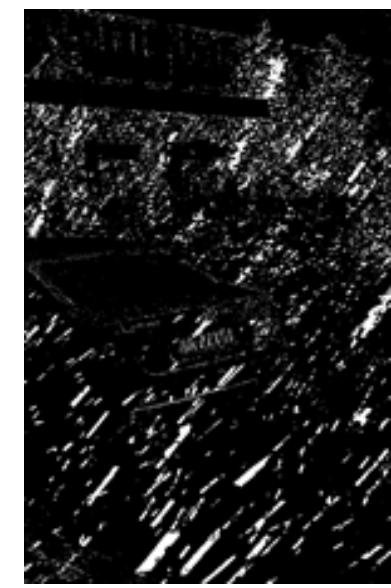

(b) frame differencing method

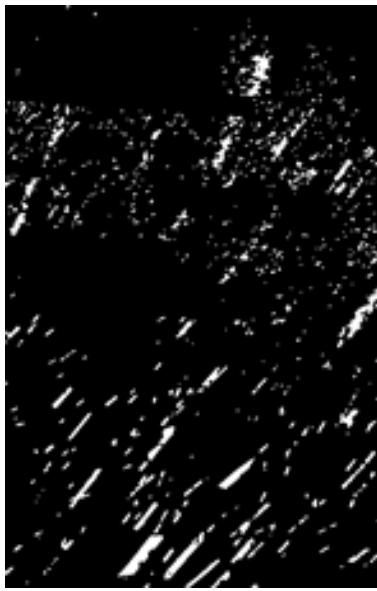

(c) presented method

Fig.4 Detection of snow in video images 


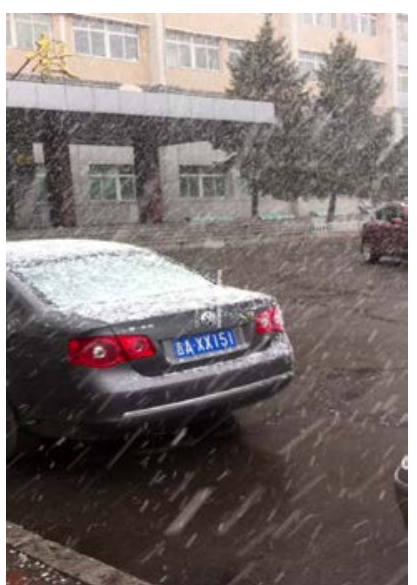

(a) original video frame

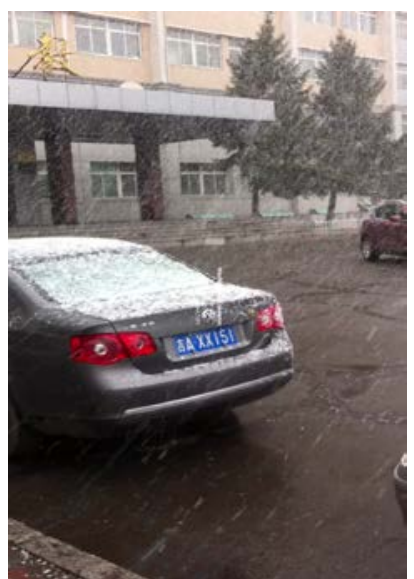

(b)snow removal frame

Fig.5 The results of snow removal

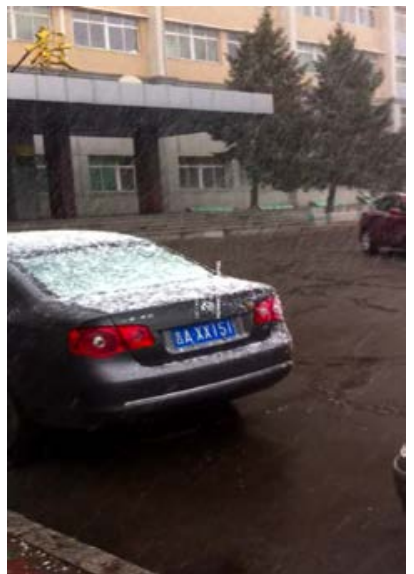

(c)defogged frame

\section{Summary}

This paper studies the snow in the video image, because of the physical characteristics and fast moving randomness, the snow video image restoration will be more difficult. Proved by experiments, the method presented in this paper can effectively detect and remove the snow in the video image, enhance the overall visual effect of video image under snow, retaining the important information, and restoration result is clear and natural, avoiding the distortion of video image. Also it can be widely used in other fast moving small objects in the natural environment, has a certain application value.

\section{Acknowledgments}

This work was supported by a grant from science and technology support program of Jilin Province (No.20140204045GX, No.20140203014GX and No. 20110355 ).

\section{References}

[1] K GARG, S K NAYAR. When does a camera see rain?[C]. IEEE International Conference on Computer Vision.2005,1067-1074.

[2] GARG K, NAYAR S K. Detection and removal of rain from videos[C]//Proceeding of Computer Society Conference on Computer Vision and Pattern Recognition. Washington D C, USA, 2004: 528-535.

[3] P BARNUM, T KANADE, S NARASIMHAN. Spation-temporal frequency analysis for removing rain and snow from videos[C].Workshop on Photometric Analysis For Computer Vision. 2007:14-21.

[4] P BARNUM, T KANADE, S NARASIMHAN. Analysis of rain and snow in frequency Space[J]. Internal Journal of Computer Vision, 2009,86 (2): 256-274.

[5] Jing Xu, Wei Zhao, Peng Liu, Xianglong Tang. Rain Removal from Video Using Dark Channel Prior and Guided Filter [J]. ICIC Express Letter.8(12):1993-1998,2012. 